\title{
The Next Step: The California Cybersecurity Institute's Anti-Trafficking Virtual Reality Immersion Training
}

\author{
Danielle Borrelli and Benjamin Thomas Greer
}

Please cite this article as: D Borrelli and B T Greer, 'The Next Step: The California Cybersecurity Institute's Anti-Trafficking Virtual Reality Immersion Training', Anti-Trafficking Review, issue 17, 2021, pp. 154-160, https://doi.org/10.14197/ atr.2012211711

Twenty years after the passage of the United States Trafficking Victims Protection Act of 2000, constructing intermediate and advanced training platforms is an uncommon endeavour by most anti-trafficking trainers and advocates. Existing 'advanced' anti-trafficking training programmes generally focus on trafficking's nexus to a particular sector of the economy, while remaining at a basic awareness level of criminal typology and crime scene complexity. With intentionality and expert planning, Virtual Reality (VR) immersion training can go further than this construction by advancing and testing the skillset of frontline and first responders (e.g., law enforcement, service providers, and prosecutors). VR provides a technological mechanism to replicate an environment through a simulation using software and specialised hardware. ${ }^{1}$ VR-based platforms have the capability to advance a multitude of learning goals and objectives by presenting users with real-life scenarios, as well as differing outcomes based on their actions. They can incorporate situated cognitive modelling scenario actions based on the pedagogy of scaffold learning. This decision-based operational learning model allows designers to create different simulations and outcomes of the same situation, thereby providing users with the opportunity to demonstrate acquired knowledgebased decision-making analyses.

A crucial feature of field or practical training is the capability of repetition without knowledge degradation. An experiential-based learning platform needs to include outcome variations based on learners' decision-making and their

1 S Ayush, et al., 'Virtual Reality: Blessings and risk assessment', arXiv preprint, 31 August 2017, retrieved 16 July 2021, https://arxiv.org/ftp/arxiv/papers/1708/ 1708.09540.pdf.

This is an open-access article distributed under the terms of the Creative Commons Attribution License (CC-BY). Under the CC-BY license, the public is free to share, adapt, and make commercial use of the work. Users must always give proper attribution to the authors and the Anti-Trafficking Review. 
correlated consequences ${ }^{2}$ such as scenarios and experiences, which are likely to occur in practice. Training scenarios implementing VR-created three-dimensional immersive storytelling conditions do not manifest in real-world consequences, and provide the ability to repeat the exercise until the participant develops the necessary competence. If built with intention and knowledge, VR-based training platforms could provide the participant learners with a rich economical alternative to costly in-person training. ${ }^{3}$ Additionally, VR experiences are meant to enhance the classroom environment and not replace foundational learning.

\section{Current Anti-Trafficking VR Training}

'Serious games' are tools of an institution but require an educator or instructor to place the game into an educational context. ${ }^{4}$ If properly designed, VR serious games facilitate creative decision-making challenges. A game-like experience can help people process scenarios or topics in more detail and highlight participants' decision-making ramifications. Emerging technologies used to communicate and elucidate suffering and raise awareness can be best termed 'humanitarian communication'. These learning modalities 'distanc[e] the spectator from the spectacle of the sufferers through [a framing device] while enabling proximity between the two through narrative and visual resources that invite our empathetic judgment towards the spectacle. ${ }^{5}$ Such distancing helps to prevent potential harm from observers, trainees, or advocates, and instead enables the development of emotions, understanding, and respect towards an individual traumatised by trafficking. This is an important feat as stereotypes and ineffective measures are often perpetuated through misinformed trainings, awareness-raising, and educational campaigns.

2 S Gabriel, 'Teaching Human Rights With Video Games?', in M Pivec and J Gründler (eds.), Proceedings of the 11th European Conference on Game-Based Learning, Academic Conferences and Publishing International Limited, Reading, 2017, pp. 191-196.

3 G Carlson and N Caporusso, 'A Physically Immersive Platform for Training Emergency Responders and Law Enforcement Officers', in S Nazir, AM Teperi, and A PolakSopińska (eds.), Advances in Human Factors in Training, Education, and Learning Sciences: Proceedings of the AHFE 2018 International Conference on Human Factors in Training, Education, and Learning Sciences, Springer, Cham, 2018, pp. 108-116, https://doi. org/10.1007/978-3-319-93882-0_11.

4 Gabriel.

5 E O'Brien and H Berents, 'Virtual Saviours: Digital games and anti-trafficking awareness-raising', Anti-Trafficking Review, issue 13, 2019, pp. 82-89, p. 84, https://doi. org/10.14197/atr.201219136. 
There are three main serious games currently used to train and raise awareness of trafficking: BAN Human Trafficking; (Un)Trafficked; and Missing: Game for a Cause. ${ }^{6}$ The programmes were launched in 2014, 2017, and 2016, respectively. Developers utilised a 'choose-your-own-adventure style of storytelling ${ }^{77}$ with limited outcomes and limited re-playability. ${ }^{8}$ Limited re-playability refers to a game's continued play value after the first initial completion; specifically, do or can the scenarios change or differ from the last iteration? The stated intention behind all three games was to raise awareness, effectively positioning these programmes as passive educative tools with narrow reach and impact. Except for (Un)Trafficked having been played more than 100,000 times, mostly by individuals in India, there is currently no publicly accessible data reporting on the players of the games and no evaluation of their impacts on the participants' awareness and understanding of human trafficking pre- and post-experience. ${ }^{?}$

The California Cybersecurity Institute (CCI) at California Polytechnic State University specialises in the application of the pedagogy 'Learn by Doing', taking real-world problems and developing solutions within a controlled, lablike environment. According to Edgar Dale's Cone of Experience, ${ }^{10}$ learners remember 10 per cent of read material, 20 per cent of heard information, and a staggering 90 per cent of information gained through experience. The CCI's Anti-Trafficking Virtual Reality Immersion Training (ATVRIT) seeks to deliver on this metric because immersive storytelling can result in enhanced learning and outcomes. Additionally, serious games are increasingly viewed as avenues to introduce humanitarian communication into the public conversation.

\section{Immersive Storytelling Is Crucial}

Story framing is a critical component of scenario-based VR learning, as it sets the background and learning borders through an in-game situation. If designed holistically, participants can be exposed to various characters beyond the suspected 'trafficker' and suspected 'victim'. There are numerous ancillary roles designers can build into the experience that mimic a real-world setting. As in other anti-

$6 \quad$ Ibid., p. 83.

7 Ibid.

8 Please note, the terminology used, such as 'games', 'choose your own adventure', or 're-playability', is part of the VR industry vernacular and not meant as a pejorative.

9 O'Brien and Berents.

10 E Dale, Audio-Visual Methods in Teaching, $3^{\text {rd }}$ Edition, Holt, Rinehart \& Winston, New York, 1969. 
trafficking VR training models, such as Radical Empathy ${ }^{11}$ or Apex Officer, ${ }^{12}$ a focus on empathy, de-escalation, and the mitigation of racial bias are at the forefront. Both platforms, individually created by other organisations, engage the senses for a deeper level of understanding and skill acquisition.

The CCI first built ATVRIT in 2019 to help law enforcement and advocacy organisations better understand the situations and experiences of trafficked individuals. Experiencing limitations with software to enhance the storytelling experience, the CCI quickly transitioned to Unity. Unity is a premier VR game creation platform, noted for providing developers with enhanced assets, customisation, and programming. In the ongoing development of ATVRIT, the programme also seeks to address perspectives that negatively impact trafficked persons and other marginalised groups. ATVRIT will always focus on addressing personal and culturally-embedded heuristics, specifically those geared towards gender, race, sexuality, nationality, and ethnicity. The provided experience will seek to engage learners in deconstructing biases based on these characteristics in order to create a more holistic representation. Another learning objective of ATVRIT is the incorporation of harm reduction methodologies related to evidence collection, including digital evidence collection.

As of now, ATVRIT participants experience the dynamic elements involved with a situation of human trafficking within an illicit massage business. The participant, also known as user or trainee, has three scenarios to choose from: an investigator, a service provider, and a bystander. Because the investigator scenario involves more complex onscreen interactions, in this paper we focus on this specific role. To start, the investigator opens the programme to a street scene, where their attention is drawn to an active investigation of a potential human trafficking situation. They immediately see a massage parlour embedded into a city landscape with neon lights, covered windows, and a list of massage prices. Upon entering the establishment, the user arrives in a small waiting room with blue walls and framed pictures of massage scenes; the room is sterile and equipped with security cameras and two small chairs. Further entry into the room reveals another door that provides access to an office. The office is minimally furnished, with a desk, security monitors showing a live feed to massage rooms, and a white board with names, monetary figures, and a hand-drawn schedule. Going back out of the room, the trainee sees a flight of stairs leading to two massage rooms. Another flight of stairs up is a kitchen, bathroom, and single bedroom with six individual beds. The next two floors have bare, blue walls with little decor; each room is clean and neatly organised. A total of six characters are in the scenario,

11 'Human Trafficking Awareness Training for Everyone', Radical Empathy, retrieved 14 June 2021, https://www.reefcares.org.

12 'About Us', Apex Officer, retrieved 14 June 2021, https://www.apexofficer.com/ about. 
each with different genders and purposes for being in the massage business. Once the user performs an initial walk-through, they are tasked with articulating what is occurring in the establishment. One of the first interactions is with a middle-aged East Asian person at the front desk, whose country of origin is yet to be discerned. Immediately, the user is challenged with how to interact with the person in front of them. They must seek and ask the right questions that will reveal why this person is in this setting. Additionally, optional questions to gather more informational context around the situation appear on screen and produce pre-programmed responses. Depending on the question asked, back-end analytics record potential biases from the user. The bias score is later revealed upon the end of the programme. On the topic of bias, the intention of this programme is not to reinforce problematic stereotypes, such as 'every massage business is involved in trafficking', but rather to explore what trafficking looks like when it does occur in such establishments. Questions proposed in ATVRIT that refocus the user on how to properly respond to a trafficking situation include, 'Is there anything I can do for you right now?'

As the participant moves through the scene, several items, characters, and evidence pieces can additionally be engaged. Users can ask questions of each character to gain a better understanding of the situation, and the conversations between the characters and the user's avatar are audible, with subtitles optional. The user interacts with evidence pieces, learning what to identify during an investigation as well as the proper collection methodologies. The user interface is equipped with tools for improving interview technique as well as evidence collection. Each evidence piece has an option for how it should be stored; items like USB flash drives, hard drives, cameras, laptops, and SD cards have additional content that illuminates or reframes the situation.

The investigator role is created as predominately static, meaning the focus is on analysing their surroundings. The limited interactions, such as asking questions and collecting evidence, are programmed with the goal of testing users' knowledge and understanding of the trafficking experience. Interactions with evidence and characters will challenge heuristics, as each character relays information and experiences framed through quantitative and qualitative understandings of the known complexities of trafficking situations. At the end of the simulation, users are presented with a score that assesses interactions with characters as well as collected or overlooked items. 


\section{Challenges: Myths, bias, and stereotypes}

To be successful, serious games require an informed educator to place the game into an educational context. ${ }^{13}$ A game-like experience can help people process scenarios or topics in more detail and highlight decision-making ramifications. ${ }^{14}$ An artificial environment provides a well-informed creator the opportunity to subconsciously address institutional or social issues like bias (e.g., framing bias, narrative fallacy, anchoring bias, or confirmation bias), narrative myths, or stereotypes. To effectively build in bias checks and the deconstruction of myths and stereotypes, the creator must first be first aware of these biases, and secondly, seek input from subject matter experts. As the next version of the ATVRIT platform is constructed, tested, and updated, the CCI has engaged trafficking experts from law enforcement, victim service providers, and professional training entities for such professional input. Specifically, the CCI will continue its relationship with the California Governor's Office of Emergency Services (Cal OES) California Specialized Training Institute (CSTI) throughout ATVRIT's development. Through the CSTI's network of trafficking experts in the fields of law, law enforcement, service provision, trauma-informed care, as well as homeland security and threat analysis, ATVRIT seeks to specifically deconstruct racial bias of victim and perpetrator ethnicity, country of origin, and gender. One option ATVRIT is considering is building virtual scenarios to not include a victim avatar or keep the victim's gender ambiguous. After participants complete the training, instructors will ask them for their opinion as to the ethnicity and gender of the victim; this educative reflection on the simulation will allow instructors to engage participants in an analytical exercise to examine how and why they made the assumptions they did. By allowing bias to be 'organically' illustrated, the impact on participants is more profound and lasting.

\section{Conclusion}

Digital gaming and virtual learning platforms will expand the boundaries of experiential-based anti-trafficking trainings. The success of virtual-based immersive trainings is directly dependent on a series of factors, including storytelling, re-playability, and supplemental in-person trainings. The California Cybersecurity Institute has accepted the challenge of designing an anti-trafficking immersion training programme that advances beyond awareness education and aims to test law enforcement and first responders' specific skillsets. This multilayered programme looks to incorporate all the concepts of 'serious gaming'

13 Gabriel.

14 Ibid. 
within a law enforcement and humanitarian communication format. Trafficking is a dynamic and fluid criminal activity. As law enforcement, academia, and victims' services organisations learn more about the tactics and typologies of trafficking, ATVRIT will revise accordingly. Moving forward, ATVRIT will continue to advance the simulation environment to better reflect the nature of trafficking situations as gleaned from subject matter experts seeking to address implicit biases and stereotypes in programming. ATVRIT programmers acknowledge the growing demand for not only effective, accurate trainings, but also for the inclusion of reflexive, harm-reducing techniques.

Danielle Borrelli, MPP is the Operations Coordinator at the California Cybersecurity Institute (CCI) and the Program Lead for the Trafficking Investigations Hub (TIH). She develops and delivers training and technical resources that address the role of technology in recruiting and exploiting victims of human trafficking. In addition, Danielle serves on the San Luis Obispo (SLO) County's District Attorney's Human Trafficking Task Force and has helped start and consulted on the development and sustainability of four separate housing programmes for trafficked persons. Email: dborrell@calpoly.edu

Benjamin Thomas Greer, J.D. is a subject matter expert in human trafficking and exploitation for the California Governor's Office of Emergency Services (Cal OES), instructing and developing trafficking courses for law enforcement and emergency management personnel. Before joining Cal OES, he served as a Special Deputy Attorney General for the California Department of Justice and helped deliver a comprehensive report entitled The State of Human Trafficking in California 2012. Aside from his work with Cal OES, he is currently attending the Naval Postgraduate School's Center for Homeland Defense and Security and is a Research Associate for the University of Cambridge's Centre for Applied Research in Human Trafficking (CCARHT). Email: benjamin.greer@caloes.ca.gov 